\title{
STUDIES ON HETEROSIS FOR YIELD IN BRINJAL (SOLANUM MELONGENA. L)
}

\section{G. KALAIYARASI, S. RANJITH RAJA RAM*, K. R. SARAVANAN}

Department of Genetics and Plant Breeding, Faculty of Agriculture, Annamalai University, Annamalai Nagar 608 o02, Tamil Nadu, India

\begin{abstract}
Heterosis breeding is one of the most efficient tools to exploit the heterotic response for various useful traits. The Line $\times$ Tester mating design was undertaken with seven lines and three testers were evaluated along with twenty one hybrids in randomized block design to estimate the magnitude of heterosis for yield and its yield contributing characters. The best way to utilize heterosis in crop is to generate $\mathrm{F}_{1}$ hybrids having maximum heterozygosity, thereby facilitating the identification and selection of hybrid vigor. The positive significant standard heterosis for fruit yield per plant was maximum with $\mathrm{L}_{3} \times \mathrm{T}_{1}(28.94 \%)$ followed by $\mathrm{L}_{7} \times \mathrm{T}_{3}(15.73 \%)$ and $\mathrm{L}_{7} \times \mathrm{T}_{1}(15.10 \%)$ respectively. Some of the promising hybrids have showed desirable heterosis for plant height, number of fruits per plant,1000 seed weight, seedling shoot length, seedling root length and seedling dry weight.
\end{abstract}

Keywords: Heterosis, parents, F1 hybrids and yield characters

\section{INTRODUCTION}

Brinjal (Solanum melongena. L) is one among the very important vegetable crop cultivating in India [1]. Brinjal is often cross pollinated and possess considerable diversity for plant types, fruit yield and yield attributing characters and thus offers an opportunity to exploit the genetic diversity for development of hybrid varieties. In ancient system of medicine, it has been used in control of many diseases, thus brinjal has medicinal properties [2]. This crop contains wide variety of dietary nutrients and minerals together with vitamins [3]. In order to obtain more varieties with high yielding and disease resistance characteristics, many attempts have been made by researchers. Exploring hybrids is one among that techniques [4]. In the present investigation, a study was conducted to study the heterosis in various crosses.

\section{MATERIALS AND METHODS}

The experiment study was carried out in Plant Breeding Farm, Department of Genetics and Plant Breeding, Faculty of Agriculture, Annamalai University, Annamalai Nagar during 2016 February-2017 January. The genotypes such as [Chidambaram Local-1 $\left(\mathrm{L}_{1}\right)$, Chidambaram Local-2 $\left(\mathrm{L}_{2}\right)$, MDU-1 $\left(\mathrm{L}_{3}\right)$, KKM-1 $\left(\mathrm{L}_{4}\right)$, Bhavani Local $\left(\mathrm{L}_{5}\right), \mathrm{CO}-1\left(\mathrm{~L}_{6}\right)$ and CO-2 $\left(\mathrm{L}_{7}\right)$ ] and three testers [Palur-1 $\left(\mathrm{T}_{1}\right)$, Palur-2 $\left(\mathrm{T}_{2}\right)$ and Annamalai $\left.\left(\mathrm{T}_{3}\right)\right]$ collected from Vegetable Research Station, Palur and Department of Genetics and Plant Breeding, Annamalai University, were used for the studies. The seeds of ten parents were sown in rows on a raised bed nursery followed by the normal nursery practices. The transplanting was done on $30 \mathrm{~d}$ old seedlings with the spacing of $75 \times 60 \mathrm{~cm}$. Ten plants per genotype were maintained. Recommended practices like of manuring, irrigation and plant protection measures were followed. The observations recorded were days to first flowering, plant height, total number of branches per plant, number of fruits per plant, fruit length, fruit girth, fruit weight, fruit yield per plant, 1000 seed weight, seed germination percentage, seedling shoot length, seedling root length and seedling dry weight.

\section{RESULTS AND DISSCUSION}

Analysis of variance for combining ability revealed that significant differences among lines and testers in respect of gca for all the characters in both the generations. The significance of $g c a$ variances thus reflected the importance of non-additive gene action for these traits. Similar results were also reported by Das and Barua [5] and Rai and Asati [6]. The differences among hybrids due to interaction between lines and testers in respect of $s c a$ were also found significant for all the characters in $F_{1}$ generation indicating the importance of non-additive gene action. Similar results of gene action for these traits were also reported by Reddy, E. E. P. and A. I. Patel (2014). The predominant role of non-additive gene action in $\mathrm{F}_{1}$ generation was observed for all the traits. For all the thirteen characters the ratio ( $G C A / \mathrm{SCA})$ value less than unity indicated the predominance of non-additive gene action in the inheritance of these traits (table 1).

Heterosis was estimated for thirteen characters and

Received 19 March 2018; Accepted 30 April 2018

*Corresponding Author

S. Ranjith Raja Ram

Department of Genetics and Plant Breeding, Faculty of Agriculture, Annamalai University, Annamalai Nagar 608002, Tamil Nadu, India

Email: ranjithplantbreeder@gmail.com

( $T$ This article is open access and licensed under the terms of the Creative Commons Attribution License (http://creativecommons.org/licenses/by/4.o/) which permits unrestricted, use, distribution and reproduction in any medium, or format for any purpose, even commercially provided the work is properly cited. Attribution - You must give appropriate credit, provide a link to the license, and indicate if changes were made. 
expressed as percentage. The first three cross of each characters for over mid parent ( $\mathrm{d}_{\mathrm{i}}$-relative heterosis), better parent ( $\mathrm{d}_{\mathrm{ii}}$-heterobeltiosis) and standard parent ( $\mathrm{d}_{\mathrm{iii}}{ }^{-}$ standard heterosis) has been mentioned below in table 2 .

\section{Days to first flowering}

The hybrid $\mathrm{L}_{2} \times \mathrm{T}_{2}$ recorded maximum significant negative relative heterosis of $\left(-9.21\right.$ per cent) followed by $\mathrm{L}_{2} \times \mathrm{T}_{3}$ recorded $\left(-3.64\right.$ per cent) and $L_{4} \times T_{1}(-2.79$ per cent) respectively. And the crosses $\mathrm{L}_{2} \times \mathrm{T}_{2}(-14.57$ per cent) followed by $\mathrm{L}_{1} \times \mathrm{T}_{1}\left(-5.07\right.$ per cent) and $\mathrm{L}_{2} \times \mathrm{T}_{3}(-4.95$ per cent) recorded negative and significant heterobeltios. The standard heterosis ranged from-18.90 for $\mathrm{L}_{2} \times \mathrm{T}_{2}$ toL $\mathrm{L}_{1} \times \mathrm{T}_{2}(-$ 2.50) and the crosses $L_{2} \times T_{2}$ recorded maximum negative standard heterosis $\left(-18.90\right.$ per cent) followed by $\mathrm{L}_{4} \times \mathrm{T}_{1}(-$ 17.77 per cent) and $L_{7} \times T_{1}(-16.38$ per cent) for this trait.

\section{Plant height (cm)}

The hybrids showed significantly negative heterosis over mid parent. The maximum negative relative heterosis was observed in $\mathrm{L}_{1} \times \mathrm{T}_{2}\left(-25.54\right.$ per cent) followed by $\mathrm{L}_{1} \times \mathrm{T}_{1}(-$ 27.12) and $L_{1} \times T_{3}(-15.62)$. The crosses $L_{1} \times T_{2}(-29.92$ per cent) followed by $L_{1} \times T_{1}(-27.55)$ per cent and $L_{1} \times T_{3}(-16.27$ per cent) combinations recorded negative and significant heterobeltiosisfor this trait. The standard heterosis ranged from $L_{1} \times T_{2}(-29.92$ per cent $)$ to $L_{5} \times T_{3}(9.63$ per cent $)$ and the crosses $\mathrm{L}_{1} \times \mathrm{T}_{2}$ recorded maximum negative standard heterosis of $\left(-29.92\right.$ per cent) followed by $L_{1} \times T_{1}(-27.55$ per cent) and $L_{1} \times T_{3}(-16.27$ per cent) respectively.

\section{Total number of branches per plants}

The cross $\mathrm{L}_{2} \times \mathrm{T}_{3}$ recorded significant positive relative heterosis (37.17 per cent) followed $\mathrm{L}_{6} \times \mathrm{T}_{3}$ (18.41 per cent) and $\mathrm{L}_{1} \times \mathrm{T}_{3}$ (8.48 per cent) respectively. Two crosses have recorded significant positive heterobeltiosis viz., $\mathrm{L}_{2} \times \mathrm{T}_{3}$ (15.13 per cent) followed by $\mathrm{L}_{6} \times \mathrm{T}_{3}$ (4.61 per cent) for this trait. And the positive significant commercial heterosis ranged from $\mathrm{L}_{3} \times \mathrm{T}_{1}$ (97.73 per cent) to $\mathrm{L}_{5} \times \mathrm{T}_{2}$ (10.21 per cent) and the crosses of $\mathrm{L}_{3} \times \mathrm{T}_{1}$ recorded maximum significant positive standard heterosis of ( 97.73 per cent) followed by $\mathrm{L}_{7} \times \mathrm{T}_{1}(87.10$ per cent $)$ and $\mathrm{L}_{4} \times \mathrm{T}_{1}(63.36$ per cent) for this trait.

\section{Number of fruits per plant}

The hybrid $\mathrm{L}_{2} \times \mathrm{T}_{3}$ recorded significant positive relative heterosis of ( 14.56 per cent) followed by $\mathrm{L}_{6} \times \mathrm{T}_{2}$ (9.38 per cent). One crosses have recorded significant positive heterobeltiosis viz., $\mathrm{L}_{2} \times \mathrm{T}_{3}(14.29$ per cent) for this trait.

The positive significant commercial heterosis ranged from $\mathrm{L}_{7} \times \mathrm{T}_{1}\left(62.29\right.$ per cent) to $\mathrm{L}_{5} \times \mathrm{T}_{2}$ (12.07 per cent) and the cross of $\mathrm{L}_{3} \times \mathrm{T}_{1}$ recorded maximum significant positive standard heterosis (77.91 per cent) followed by $\mathrm{L}_{2} \times \mathrm{T}_{3}$ (74.11 per cent) and $\mathrm{L}_{7} \times \mathrm{T}_{1}$ (62.29 per cent) respectively.

\section{Fruit length (cm)}

The hybrid $\mathrm{L}_{1} \times \mathrm{T}_{1}$ exhibited significant positive relative heterosis (21.81 per cent) followed by $\mathrm{L}_{4} \times \mathrm{T}_{1}(17.76$ per cent) and $\mathrm{L}_{3} \times \mathrm{T}_{1}$ (13.27 per cent) respectively. The crosses have recorded significant positive heterobeltiosis are $\mathrm{L}_{1} \times$ $\mathrm{T}_{1}$ (15.74 per cent) followed by $\mathrm{L}_{3} \times \mathrm{T}_{1}$ (8.21 per cent), $\mathrm{L}_{4} \times$ $\mathrm{T}_{1}$ (4.38 per cent) for this trait.

The negative significant commercial heterosis was ranging from $\mathrm{L}_{1} \times \mathrm{T}_{1}(28.54$ per cent $)$ to $\mathrm{L}_{2} \times \mathrm{T}_{2}(4.25$ per cent $)$ and the cross $\mathrm{L}_{1} \times \mathrm{T}_{1}(28.54$ per cent) recorded maximum significant positive standard heterosis followed by $\mathrm{L}_{1} \times \mathrm{T}_{2}$ (20.79 per cent) and $\mathrm{L}_{3} \times \mathrm{T}_{1}(20.18$ per cent) for this trait.

\section{Fruit girth}

The hybrid $L_{1} \times T_{1}$ exhibited significant positive relative heterosis (29.05 per cent) followed by $\mathrm{L}_{1} \times \mathrm{T}_{3}$ (14.75 per cent) and $\mathrm{L}_{3} \times \mathrm{T}_{1}$ (11.83 per cent) respectively. One cross has recorded significant positive heterobeltiosis viz., $\mathrm{L}_{3} \times$ $\mathrm{T}_{1}$ (11.47 per cent) respectively. And positive significant commercial heterosis and it was ranging from $\mathrm{L}_{3} \times \mathrm{T}_{1}$ (102.76 per cent) to $\mathrm{L}_{6} \times \mathrm{T}_{1}(9.84$ per cent) and the hybrid $\mathrm{L}_{3} \times \mathrm{T}_{1}$ recorded maximum significant positive standard heterosis of (102.76 per cent) followed by $\mathrm{L}_{3} \times \mathrm{T}_{2}(94.49$ per cent) and $L_{1} \times T_{1}(81.89 p e r$ cent) for this trait.

\section{Fruit weight}

The hybrid $\mathrm{L}_{4} \times \mathrm{T}_{3}$ exhibited significant negative relative heterosis (105.96 per cent) followed by $\mathrm{L}_{7} \times \mathrm{T}_{3}$ (88.30 per cent) and $L_{1} \times T_{1}(-81.33$ per cent) respectively. The crosses have recorded significant positive heterobeltiosis viz., $\mathrm{L}_{4} \times \mathrm{T}_{3}$ (82.62 per cent) followed by $\mathrm{L}_{7} \times \mathrm{T}_{3}\left(68.29\right.$ per cent) and $\mathrm{L}_{6} \times$ $\mathrm{T}_{3}$ (64.31 per cent) for this trait. And the crosses recorded positive significant commercial heterosis and it was ranging from $\mathrm{L}_{1} \times \mathrm{T}_{1}\left(120.81\right.$ per cent) to $\mathrm{L}_{2} \times \mathrm{T}_{2}(8.71$ per cent) and the hybrid $\mathrm{L}_{1} \times \mathrm{T}_{1}$ recorded maximum significant positive standard heterosis (120.81per cent) followed by $\mathrm{L}_{5} \times \mathrm{T}_{1}$ (104.88 per cent) and $\mathrm{L}_{7} \times \mathrm{T}_{2}$ (92.36 per cent) respectively.

\section{Fruit yield per plant}

The hybrids namely $\mathrm{L}_{5} \times \mathrm{T}_{2}$ recorded (84.09 per cent) followed by $\mathrm{L}_{2} \times \mathrm{T}_{2}$ (46.43 per cent) and $\mathrm{L}_{2} \times \mathrm{T}_{3}$ (17.67 per cent) respectively. The hybrids recorded significant positive heterobeltiosis viz., $\mathrm{L}_{5} \times \mathrm{T}_{2}$ (77.90 per cent) followed by $\mathrm{L}_{2} \times$ $\mathrm{T}_{2}$ (40.03 per cent) for this trait. And standard heterosis for this trait and it was ranging from $\mathrm{L}_{3} \times \mathrm{T}_{1}$ (28.94 per cent) to $\mathrm{L}_{5} \times \mathrm{T}_{2}$ (9.75 per cent) and it was maximum with the hybrid $\mathrm{L}_{3} \times \mathrm{T}_{1}$ (28.94 per cent) followed by $\mathrm{L}_{7} \times \mathrm{T}_{3}(15.78$ per cent) and $\mathrm{L}_{7} \times \mathrm{T}_{1}(15.10$ per cent) for this trait.

\section{0 seed weight}

The hybrid $\mathrm{L}_{2} \times \mathrm{T}_{2}$ recorded significant positive relative heterosis of (49.94 per cent) followed by $\mathrm{L}_{6} \times \mathrm{T}_{1}(21.88 \mathrm{per}$ cent) and $\mathrm{L}_{1} \times \mathrm{T}_{2}$ (20.44 per cent) respectively. One out of twenty one crosses have recorded significant positive heterobeltiosis namely, $\mathrm{L}_{2} \times \mathrm{T}_{2}(28.25$ per cent $)$ for this trait. The crosses recorded positive significant commercial heterosis and it was ranging from $\mathrm{L}_{7} \times \mathrm{T}_{1}(72.15$ per cent) to $\mathrm{L}_{7} \times \mathrm{T}_{2}$ (18.99 per cent) and the cross of $\mathrm{L}_{7} \times \mathrm{T}_{1}$ recorded maximum significant positive standard heterosis of (72.15per cent) followed by $\mathrm{L}_{6} \times \mathrm{T}_{1}(56.20$ per cent $)$ and $\mathrm{L}_{3} \times$ $\mathrm{T}_{1}$ (38.86 per cent) for this trait.

\section{Seed germination percentage}

The hybrid $\mathrm{L}_{3} \times \mathrm{T}_{1}$ exhibited significant positive relative heterosis of (5.27 per cent) followed by $\mathrm{L}_{7} \times \mathrm{T}_{1}$ (2.86 per cent) and $\mathrm{L}_{3} \times \mathrm{T}_{2}$ (2.14 per cent) respectively. And the crosses have recorded significant positive heterobeltiosis viz., $\mathrm{L}_{3} \times \mathrm{T}_{1}$ (3.58 per cent) followed by $\mathrm{L}_{2} \times \mathrm{T}_{2}(1.23 \mathrm{per}$ cent) and $\mathrm{L}_{7} \times \mathrm{T}_{1}\left(1.12\right.$ per cent) respectively. The hybrid $\mathrm{L}_{3}$ $\times \mathrm{T}_{1}$ recorded maximum significant positive standard heterosis of (11.91 per cent) followed by $\mathrm{L}_{7} \times \mathrm{T}_{1}(9.45 \mathrm{per}$ cent) and $\mathrm{L}_{3} \times \mathrm{T}_{2}$ (8.05 per cent) for this character.

\section{Seedling shoot length}

The hybrid $\mathrm{L}_{7} \times \mathrm{T}_{1}$ exhibited significant positive relative heterosis of (30.16per cent) followed by $\mathrm{L}_{5} \times \mathrm{T}_{1}$ (24.37per cent) and $\mathrm{L}_{4} \times \mathrm{T}_{1}(23.57$ per cent) respectively. The crosses have recorded significant positive heterobeltiosis viz., $\mathrm{L}_{5} \times$ $\mathrm{T}_{1}$ (23.63 per cent) followed by $\mathrm{L}_{7} \times \mathrm{T}_{1}(23.34$ per cent $)$ and $\mathrm{L}_{4} \times \mathrm{T}_{1}(22.84$ per cent $)$ respectively. 
And the crosses recorded positive significant commercial heterosis and it was ranging from $\mathrm{L}_{7} \times \mathrm{T}_{1}(21.58$ per cent) to $\mathrm{L}_{1} \times \mathrm{T}_{2}$ (8.79 per cent) and the hybrid $\mathrm{L}_{7} \times \mathrm{T}_{1}$ recorded maximum significant positive standard heterosis of (21.58 per cent) followed by $\mathrm{L}_{6} \times \mathrm{T}_{1}\left(19.49\right.$ per cent) and $\mathrm{L}_{7} \times \mathrm{T}_{3}$ (13.15 per cent) for this character.

\section{Seedling root length}

The hybrid $\mathrm{L}_{1} \times \mathrm{T}_{3}$ exhibited significant positive relative heterosis of ( 59.54 per cent) followed by $\mathrm{L}_{2} \times \mathrm{T}_{3}$ (40.62per cent) and $\mathrm{L}_{1} \times \mathrm{T}_{1}(25.23$ per cent) respectively.

The crosses have recorded significant positive heterobeltiosis viz., $\mathrm{L}_{1} \times \mathrm{T}_{3}$ (58.72 per cent) followed by $\mathrm{L}_{2}$ $\times \mathrm{T}_{3}$ (39.36 per cent) and $\mathrm{L}_{1} \times \mathrm{T}_{1}(23.45$ per cent $)$ respectively. And for commercial heterosis it was ranging from $\mathrm{L}_{6} \times \mathrm{T}_{2}\left(65.80\right.$ per cent) to $\mathrm{L}_{1} \times \mathrm{T}_{1}(23.45$ per cent). The hybrid of $\mathrm{L}_{6} \times \mathrm{T}_{2}$ recorded maximum significant positive standard heterosis of (65.80 per cent) followed by $\mathrm{L}_{7} \times \mathrm{T}_{1}$ (60.49per cent) and $\mathrm{L}_{1} \times \mathrm{T}_{3}$ (60.36 per cent) was given for this trait.

\section{Seedling dry weight}

The hybrid $\mathrm{L}_{2} \times \mathrm{T}_{2}$ exhibited significant positive relative heterosis (87.94 per cent) followed $\mathrm{L}_{5} \times \mathrm{T}_{3}$ (64.58 per cent) and $\mathrm{L}_{5} \times \mathrm{T}_{2}$ (56.10 per cent) respectively. And the crosses have recorded significant positive heterobeltiosis viz., $\mathrm{L}_{2} \times$ $\mathrm{T}_{2}$ (34.53 per cent) followed by $\mathrm{L}_{5} \times \mathrm{T}_{3}(25.40$ per cent $)$ and $\mathrm{L}_{5} \times \mathrm{T}_{2}$ (15.11 per cent) respectively. And the crosses recorded positive significant commercial heterosis and it was ranging from $L_{3} \times T_{1}\left(62.50\right.$ per cent) to $L_{1} \times T_{3}(14.71$ per cent) and the hybrid $\mathrm{L}_{3} \times \mathrm{T}_{1}$ recorded maximum significant positive standard heterosis (62.50 per cent) followed by $\mathrm{L}_{2} \times \mathrm{T}_{2}\left(37.50\right.$ per cent) and $\mathrm{L}_{5} \times \mathrm{T}_{2}(17.65$ per cent) for this character.

Table 1: Analysis of variance for thirteen characters in brinjal

\begin{tabular}{|c|c|c|c|c|c|c|c|c|c|c|c|c|c|c|}
\hline Source & df & $\begin{array}{c}\text { Days to } \\
\text { first } \\
\text { flowering }\end{array}$ & $\begin{array}{l}\text { Plant } \\
\text { height }\end{array}$ & $\begin{array}{c}\text { Total } \\
\text { number } \\
\text { of } \\
\text { branches } \\
\text { per } \\
\text { plant } \\
\end{array}$ & $\begin{array}{c}\text { Number } \\
\text { of fruits } \\
\text { per } \\
\text { plant }\end{array}$ & $\begin{array}{l}\text { Fruit } \\
\text { length }\end{array}$ & $\begin{array}{l}\text { Fruit } \\
\text { girth }\end{array}$ & $\begin{array}{c}\text { Fruit } \\
\text { weight }\end{array}$ & $\begin{array}{c}\text { Fruit yield } \\
\text { per plant }\end{array}$ & $\begin{array}{c}1000 \\
\text { seed } \\
\text { weight }\end{array}$ & $\begin{array}{c}\text { Seed } \\
\text { germination } \\
\text { percentage }\end{array}$ & $\begin{array}{c}\text { Seedling } \\
\text { shoot } \\
\text { length }\end{array}$ & $\begin{array}{c}\text { Seedling } \\
\text { root } \\
\text { length }\end{array}$ & $\begin{array}{c}\text { Seedling } \\
\text { dry } \\
\text { weight }\end{array}$ \\
\hline & & \multicolumn{13}{|c|}{ Mean Sum of Square } \\
\hline Replication & 2 & 36.49 & 2109.71 & 30.16 & 30.01 & 3.0897 & 32.70 & 625.38 & 165305.80 & 29.5660 & 28.15 & 28.75 & 29.94 & 0.24 \\
\hline Lines & 6 & $146.79 * *$ & $623.6186^{* *}$ & $26.53^{* *}$ & $30.02 * *$ & $4.1004 * *$ & $62.28^{* *}$ & 1190.77 & 44694.1 & 1.0765 & $51.28 * *$ & $3.9019 * *$ & $1.2722 * *$ & $0.03 * *$ \\
\hline Testers & 2 & $39.31^{* *}$ & $280.1652 * *$ & $45.57^{* * *}$ & $13.67^{* *}$ & 0.2372 & 13.43 & 2114.36 & 5152.6667 & 0.7020 & $104.73 * *$ & 3.3044 & 0.7799 & 0.007 \\
\hline Error & 60 & 0.11 & 7.9310 & 0.03 & 0.07 & 0.0337 & 0.056 & 3.76 & 698.2100 & 0.0611 & 0.09 & 0.0315 & 0.0939 & 0.0008 \\
\hline$G C A$ & - & -0.0884 & 4.2526 & 0.2262 & 0.1875 & 0.0194 & 0.4261 & 1.8755 & -1204.2450 & -0.0050 & 0.5398 & 0.0207 & 0.0007 & -0.0002 \\
\hline SCA & - & 42.7665 & 40.5257 & 3.1820 & 2.6220 & 0.4133 & 3.0380 & 412.6168 & 53770.3281 & 0.4669 & 4.2448 & 0.5773 & 0.3290 & 0.0159 \\
\hline GCA/SCA & - & 0.00020 & 0.10493 & 0.07108 & 0.071510 & 0.04693 & 0.14025 & 0.004545 & 0.02239 & 0.01070 & 0.12716 & 0.03585 & 0.00212 & 0.0125 \\
\hline
\end{tabular}

***: Significant at 1 percent and 5 per cent level

Table 2: Heterosis for the first three best hybrids in relative heterosis, heterobeltiosis and standard heterosis

\begin{tabular}{|c|c|c|c|c|c|c|c|c|c|c|c|c|c|}
\hline CHARACTERS & 1 & 2 & 3 & 4 & 5 & 6 & 7 & 8 & 9 & 10 & 11 & 12 & 13 \\
\hline \multirow{3}{*}{$\begin{array}{l}\text { Relative } \\
\text { Heterosis } \\
\text { (di) }\end{array}$} & $\begin{array}{c}\mathrm{L}_{2} \times \mathrm{T}_{2} \\
(9.21 \%)\end{array}$ & $\begin{array}{c}\mathrm{L}_{1} \times \mathrm{T}_{2} \\
(-25.54 \%)\end{array}$ & $\begin{array}{c}\mathrm{L}_{2} \times \mathrm{T}_{3} \\
(37.17 \%)\end{array}$ & $\begin{array}{c}\mathrm{L}_{2} \times \mathrm{T}_{3} \\
(14.56 \%)\end{array}$ & $\begin{array}{c}\mathrm{L}_{1} \times \mathrm{T}_{1} \\
(21.81 \%)\end{array}$ & $\begin{array}{c}\mathrm{L}_{1} \times \mathrm{T}_{1} \\
(29.05 \%)\end{array}$ & $\begin{array}{c}\mathrm{L}_{4} \times \mathrm{T}_{3} \\
(105.96 \%)\end{array}$ & $\begin{array}{c}\mathrm{L}_{5} \times \mathrm{T}_{2} \\
(84.09 \%)\end{array}$ & $\begin{array}{c}\mathrm{L}_{2} \times \mathrm{T}_{2} \\
(49.94 \%)\end{array}$ & $\begin{array}{c}\mathrm{L}_{3} \times \mathrm{T}_{1} \\
(5.27 \%)\end{array}$ & $\begin{array}{c}\mathrm{L}_{7} \times \mathrm{T}_{1} \\
(30.16 \%)\end{array}$ & $\begin{array}{c}\mathrm{L}_{1} \times \mathrm{T}_{3} \\
(59.54 \%)\end{array}$ & $\begin{array}{c}\mathrm{L}_{2} \times \mathrm{T}_{2} \\
(87.94 \%)\end{array}$ \\
\hline & $\begin{array}{c}\mathrm{L}_{2} \times \mathrm{T}_{3} \\
(3.64 \%)\end{array}$ & $\begin{array}{c}\mathrm{L}_{1} \times \mathrm{T}_{1} \\
(-27.12 \%)\end{array}$ & $\begin{array}{c}\mathrm{L}_{6} \times \mathrm{T}_{3} \\
(18.41 \%)\end{array}$ & $\begin{array}{c}\mathrm{L}_{6} \times \mathrm{T}_{2} \\
(9.38 \%)\end{array}$ & $\begin{array}{c}\mathrm{L}_{4} \times \mathrm{T}_{1} \\
(17.76 \%)\end{array}$ & $\begin{array}{c}\mathrm{L}_{1} \times \mathrm{T}_{3} \\
(14.75 \%)\end{array}$ & $\begin{array}{c}\mathrm{L}_{7} \times \mathrm{T}_{3} \\
(88.30 \%)\end{array}$ & $\begin{array}{c}\mathrm{L}_{2} \times \mathrm{T}_{2} \\
(46.43 \%)\end{array}$ & $\begin{array}{c}\mathrm{L}_{6} \times \mathrm{T}_{1} \\
(21.88 \%)\end{array}$ & $\begin{array}{c}\mathrm{L}_{7} \times \mathrm{T}_{1} \\
(2.86 \%)\end{array}$ & $\begin{array}{c}\mathrm{L}_{5} \times \mathrm{T}_{1} \\
(24.37 \%)\end{array}$ & $\begin{array}{c}\mathrm{L}_{2} \times \mathrm{T}_{3} \\
(40.62 \%)\end{array}$ & $\begin{array}{c}\mathrm{L}_{5} \times \mathrm{T}_{3} \\
(64.58 \%)\end{array}$ \\
\hline & $\begin{array}{c}\mathrm{L}_{4} \times \mathrm{T}_{1} \\
(-2.79 \%)\end{array}$ & $\begin{array}{c}\mathrm{L}_{1} \times \mathrm{T}_{3} \\
(-15.16 \%)\end{array}$ & $\begin{array}{c}\mathrm{L}_{1} \times \mathrm{T}_{3} \\
(8.48 \%)\end{array}$ & --- & $\begin{array}{c}\mathrm{L}_{3} \times \mathrm{T}_{1} \\
(13.27 \%)\end{array}$ & $\begin{array}{c}\mathrm{L}_{3} \times \mathrm{T}_{1} \\
(11.83 \%)\end{array}$ & $\begin{array}{c}\mathrm{L}_{1} \times \mathrm{T}_{1} \\
(-81.33 \%)\end{array}$ & $\begin{array}{c}\mathrm{L}_{2} \times \mathrm{T}_{3} \\
(17.67 \%)\end{array}$ & $\begin{array}{c}\mathrm{L}_{1} \times \mathrm{T}_{2} \\
(20.44 \%)\end{array}$ & $\begin{array}{c}\mathrm{L}_{3} \times \mathrm{T}_{2} \\
(2.14 \%)\end{array}$ & $\begin{array}{c}\mathrm{L}_{4} \times \mathrm{T}_{1} \\
(23.57 \%)\end{array}$ & $\begin{array}{c}\mathrm{L}_{1} \times \mathrm{T}_{1} \\
(25.23 \%)\end{array}$ & $\begin{array}{c}\mathrm{L}_{5} \times \mathrm{T}_{2} \\
(56.10 \%)\end{array}$ \\
\hline \multirow{3}{*}{$\begin{array}{l}\text { Heterobeltiosis } \\
\text { (dii) }\end{array}$} & $\begin{array}{c}\mathrm{L}_{2} \times \mathrm{T}_{2} \\
(-14.57 \%)\end{array}$ & $\begin{array}{c}\mathrm{L}_{1} \times \mathrm{T}_{2} \\
(-29.92 \%)\end{array}$ & $\begin{array}{c}\mathrm{L}_{2} \times \mathrm{T}_{3} \\
(15.13 \%)\end{array}$ & $\begin{array}{c}\mathrm{L}_{2} \times \mathrm{T}_{3} \\
(14.29 \%)\end{array}$ & $\begin{array}{c}\mathrm{L}_{1} \times \mathrm{T}_{1} \\
(15.74 \%)\end{array}$ & $\begin{array}{c}\mathrm{L}_{3} \times \mathrm{T}_{1} \\
(11.47 \%)\end{array}$ & $\begin{array}{c}\mathrm{L}_{4} \times \mathrm{T}_{3} \\
(82.62 \%)\end{array}$ & $\begin{array}{c}\mathrm{L}_{5} \times \mathrm{T}_{2} \\
(77.90 \%)\end{array}$ & $\begin{array}{c}\mathrm{L}_{2} \times \mathrm{T}_{2} \\
(28.25 \%)\end{array}$ & $\begin{array}{c}\mathrm{L}_{3} \times \mathrm{T}_{1} \\
(3.58 \%)\end{array}$ & $\begin{array}{c}\mathrm{L}_{5} \times \mathrm{T}_{1} \\
(23.63 \%)\end{array}$ & $\begin{array}{c}\mathrm{L}_{1} \times \mathrm{T}_{3} \\
(58.72 \%)\end{array}$ & $\begin{array}{c}\mathrm{L}_{2} \times \mathrm{T}_{2} \\
(34.53 \%)\end{array}$ \\
\hline & $\begin{array}{c}\mathrm{L}_{1} \times \mathrm{T}_{1} \\
(-5.07 \%)\end{array}$ & $\begin{array}{c}\mathrm{L}_{1} \times \mathrm{T}_{1} \\
(-27.55 \%)\end{array}$ & $\begin{array}{c}\mathrm{L}_{6} \times \mathrm{T}_{3} \\
(4.61 \%)\end{array}$ & & $\begin{array}{c}\mathrm{L}_{3} \times \mathrm{T}_{1} \\
(8.21 \%)\end{array}$ & ---- & $\begin{array}{c}\mathrm{L}_{7} \times \mathrm{T}_{3} \\
(68.29 \%)\end{array}$ & $\begin{array}{c}\mathrm{L}_{2} \times \mathrm{T}_{2} \\
(40.03 \%)\end{array}$ & ----- & $\begin{array}{l}\mathrm{L}_{2} \times \mathrm{T}_{2} \\
(1.23 \%)\end{array}$ & $\begin{array}{c}\mathrm{L}_{7} \times \mathrm{T}_{1} \\
(23.34 \%)\end{array}$ & $\begin{array}{c}\mathrm{L}_{2} \times \mathrm{T}_{3} \\
(39.36 \%)\end{array}$ & $\begin{array}{c}\mathrm{L}_{5} \times \mathrm{T}_{3} \\
(25.40 \%)\end{array}$ \\
\hline & $\begin{array}{c}\mathrm{L}_{2} \times \mathrm{T}_{3} \\
(-4.95 \%)\end{array}$ & $\begin{array}{c}\mathrm{L}_{1} \times \mathrm{T}_{3} \\
(-16.27 \%)\end{array}$ & - & -.---.-- & $\begin{array}{c}\mathrm{L}_{4} \times \mathrm{T}_{1} \\
(4.38 \%)\end{array}$ & ------ & $\begin{array}{c}\mathrm{L}_{6} \times \mathrm{T}_{3} \\
(64.31 \%)\end{array}$ & --- & ------ & $\begin{array}{c}\mathrm{L}_{7} \times \mathrm{T}_{1} \\
(1.12 \%)\end{array}$ & $\begin{array}{c}\mathrm{L}_{4} \times \mathrm{T}_{1} \\
(22.84 \%)\end{array}$ & $\begin{array}{c}\mathrm{L}_{1} \times \mathrm{T}_{1} \\
(23.45 \%)\end{array}$ & $\begin{array}{c}\mathrm{L}_{5} \times \mathrm{T}_{2} \\
(15.11 \%)\end{array}$ \\
\hline \multirow{3}{*}{$\begin{array}{c}\text { Standard } \\
\text { Heterosis } \\
\text { (diii) }\end{array}$} & $\begin{array}{c}\mathrm{L}_{2} \times \mathrm{T}_{2} \\
(-18.90 \%)\end{array}$ & $\begin{array}{c}\mathrm{L}_{1} \times \mathrm{T}_{2} \\
(-29.92 \%)\end{array}$ & $\begin{array}{c}\mathrm{L}_{3} \times \mathrm{T}_{1} \\
(97.73 \%)\end{array}$ & $\begin{array}{c}\mathrm{L}_{3} \times \mathrm{T}_{1} \\
(77.91 \%)\end{array}$ & $\begin{array}{c}\mathrm{L}_{1} \times \mathrm{T}_{1} \\
(28.54 \%)\end{array}$ & $\begin{array}{c}\mathrm{L}_{3} \times \mathrm{T}_{1} \\
(102.76 \%)\end{array}$ & $\begin{array}{c}\mathrm{L}_{1} \times \mathrm{T}_{1} \\
(120.81 \%)\end{array}$ & $\begin{array}{c}\mathrm{L}_{3} \times \mathrm{T}_{1} \\
(28.94 \%)\end{array}$ & $\begin{array}{c}\mathrm{L}_{7} \times \mathrm{T}_{1} \\
(72.15 \%)\end{array}$ & $\begin{array}{c}\mathrm{L}_{3} \times \mathrm{T}_{1} \\
(11.91 \%)\end{array}$ & $\begin{array}{c}\mathrm{L}_{7} \times \mathrm{T}_{1} \\
(21.58 \%\end{array}$ & $\begin{array}{c}\mathrm{L}_{6} \times \mathrm{T}_{1} \\
(65.80 \%)\end{array}$ & $\begin{array}{c}\mathrm{L}_{3} \times \mathrm{T}_{1} \\
(62.50 \%)\end{array}$ \\
\hline & $\begin{array}{c}\mathrm{L}_{4} \times \mathrm{T}_{1} \\
(-17.77 \%)\end{array}$ & $\begin{array}{c}\mathrm{L}_{1} \times \mathrm{T}_{1} \\
(-27.55 \%)\end{array}$ & $\begin{array}{c}\mathrm{L}_{7} \times \mathrm{T}_{1} \\
(87.10 \%)\end{array}$ & $\begin{array}{c}\mathrm{L}_{2} \times \mathrm{T}_{3} \\
(74.11 \%)\end{array}$ & $\begin{array}{c}\mathrm{L}_{1} \times \mathrm{T}_{2} \\
(20.79 \%)\end{array}$ & $\begin{array}{c}\mathrm{L}_{3} \times \mathrm{T}_{2} \\
(94.49 \%)\end{array}$ & $\begin{array}{c}\mathrm{L}_{5} \times \mathrm{T}_{1} \\
(104.88 \%)\end{array}$ & $\begin{array}{c}\mathrm{L}_{7} \times \mathrm{T}_{3} \\
(15.78 \%)\end{array}$ & $\begin{array}{c}\mathrm{L}_{6} \times \mathrm{T}_{1} \\
(56.20 \%)\end{array}$ & $\begin{array}{c}\mathrm{L}_{7} \times \mathrm{T}_{1} \\
(9.45 \%)\end{array}$ & $\begin{array}{c}\mathrm{L}_{6} \times \mathrm{T}_{1} \\
(19.49 \%)\end{array}$ & $\begin{array}{c}\mathrm{L}_{7} \times \mathrm{T}_{1} \\
(60.49 \%)\end{array}$ & $\begin{array}{c}\mathrm{L}_{2} \times \mathrm{T}_{2} \\
(37.50 \%)\end{array}$ \\
\hline & $\begin{array}{c}\mathrm{L}_{7} \times \mathrm{T}_{1} \\
(-16.38 \%)\end{array}$ & $\begin{array}{c}\mathrm{L}_{1} \times \mathrm{T}_{3} \\
(-16.27 \%)\end{array}$ & $\begin{array}{c}\mathrm{L}_{4} \times \mathrm{T}_{1} \\
(62.29 \%)\end{array}$ & $\begin{array}{c}\mathrm{L}_{7} \times \mathrm{T}_{1} \\
(62.29 \%)\end{array}$ & $\begin{array}{c}\mathrm{L}_{3} \times \mathrm{T}_{1} \\
(20.18 \%)\end{array}$ & $\begin{array}{c}\mathrm{L}_{1} \times \mathrm{T}_{1} \\
(81.89 \%)\end{array}$ & $\begin{array}{c}\mathrm{L}_{7} \times \mathrm{T}_{2} \\
(92.36 \%)\end{array}$ & $\begin{array}{c}\mathrm{L}_{7} \times \mathrm{T}_{1} \\
(15.10 \%)\end{array}$ & $\begin{array}{c}\mathrm{L}_{3} \times \mathrm{T}_{1} \\
(38.86 \%)\end{array}$ & $\begin{array}{c}\mathrm{L}_{3} \times \mathrm{T}_{2} \\
(38.86 \%)\end{array}$ & $\begin{array}{c}\mathrm{L}_{7} \times \mathrm{T}_{3} \\
(13.15 \%)\end{array}$ & $\begin{array}{c}\mathrm{L}_{1} \times \mathrm{T}_{3} \\
(60.36 \%)\end{array}$ & $\begin{array}{c}\mathrm{L}_{5} \times \mathrm{T}_{2} \\
(17.65 \%)\end{array}$ \\
\hline
\end{tabular}

\section{CONCLUSION}

The hybrids $\mathrm{L}_{3} \times \mathrm{T}_{1}, \mathrm{~L}_{7} \times \mathrm{T}_{3}$ and $\mathrm{L}_{7} \times \mathrm{T}_{1}$ recorded as superior hybrids based on the magnitude of standard heterosis for fruit yield per plant. The crosses $\mathrm{L}_{2} \times \mathrm{T}_{2}, \mathrm{~L}_{4} \times \mathrm{T}_{1}$ and $\mathrm{L}_{7} \times \mathrm{T}_{1}$ recorded for days to first flowering based on the magnitude of standard heterosis. Hybrids such as $\mathrm{L}_{7} \times \mathrm{T}_{1}$, $\mathrm{L}_{6} \times \mathrm{T}_{1}$ and $\mathrm{L}_{3} \times \mathrm{T}_{1}$ for 1000 seed weight, the crosses $\mathrm{L}_{3} \times \mathrm{T}_{1}$,
$\mathrm{L}_{7} \times \mathrm{T}_{1}$ and $\mathrm{L}_{3} \times \mathrm{T}_{2}$ for seed germination percentage, the crosses $\mathrm{L}_{7} \times \mathrm{T}_{1}, \mathrm{~L}_{6} \times \mathrm{T}_{1}$ and $\mathrm{L}_{7} \times \mathrm{T}_{2}$ for seedling shoot length and the crosses $\mathrm{L}_{6} \times \mathrm{T}_{2}, \mathrm{~L}_{7} \times \mathrm{T}_{1}$ and $\mathrm{L}_{1} \times \mathrm{T}_{3}$ for seedling root length. The hybrid $\mathrm{L}_{3} \times \mathrm{T}_{1}$ was identified as the best hybrid since it had significant standard heterosis for all the traits. The next best hybrids were $\mathrm{L}_{7} \times \mathrm{T}_{1}$ and $\mathrm{L}_{7} \times \mathrm{T}_{3}$ since they possessed desirable standard heterosis for major traits. Our results are in agreement with previous studies [7-11]. 


\section{REFERENCES}

1. Sękara A, Cebula S, Kunicki E. Cultivated eggplantsorigin, breeding objectives and genetic resources, a review. Folia Horticulturae. 2007;19:97-114.

2. Guimarães PR, Galvão AM, Batista CM, Azevedo GS, Oliveira RD, Lamounier RP, Freire N, Barros AM, Sakurai E, Oliveira JP, Vieira EC. Eggplant (Solanum melongena) infusion has a modest and transitory effect on hypercholesterolemic subjects. Brazilian Journal of Medical and Biological Research. 2000 Sep; 33:1027-36.

3. Hedges LJ, Lister CE. Nutritional attributes of spinach, silver beet and eggplant. Crop Food Res Confidential Rep. 2007 Jun; 1928.

4. Bavage, M. S., M. B. Madalageri, V. D. Gasti and D. Barman. 2006. Per se performance and magnitude of heterosis in round fruited brinjal (Solanum melongena L.) Paper presented at national seminar on appropriate technology for sustainable horticulture, Department of Horticulture, Annamalai University, Annamalai Nagar 20-21:p.5.

5. Das, G. and N. S. Barua. 2001. Heterosis and combining ability for yieldand its components in brinjal. Ann. Agric. Res., 23: 399-403.
6. Rai, N. and B. S. Asati. 2011. Combining ability and gene action studies for fruit yield and yield contributing traits in brinjal. Ind. J. Hort., 68: 212215.

7. Nalini, A., S. A. Dharwad, Patil and P. M. Salimath. 2011. Heterosis and combining ability analysis for productivity traits in brinjal (Solanum melongena L.) Karnataka J. Agric. Sci., 24: 622-625.

8. Prabhu, M., S. Natarajan and L. Pugalendhi. 2005. Studies on heterosis and mean performance in brinjal (Solanum melongena L.) Veg. Sci., 32: 86-87.

9. Reddy, E. E. P. and A. I. Patel. 2014(b). Heterosis studies for yield and yield attributing characters in brinjal (Solanum melongena L.) Sch. J. Agri. Sci., 4: 109-112.

10. Shafeeq, A., K. Madhusudan, R. R. Hanchinal, A. G. Vijayakumar and P. M. Salimath.2007. Heterosis in brinjal. Karnataka J. Agric. Sci., 20: 33-40.

11. Suneetha, Y., K. B. Kathiria, J. S. Patel and T. Srinivas. 2008. Studies on heterosis and combining ability in late summer brinjal. Ind. J. Agric. Res., 42: 171-176. 